\title{
Behandlung und Prävention mit Rifaximin
}

\begin{abstract}
Auf Reisen erworbene Diarrhöen sind weltweit ein häufiges Problem. Die Therapie ist in der Regel symptomatisch Flüssigkeitssubstitution und Antidiarrhoika. In schweren Verläufen erfolgt eine antibiotische Therapie, um die Symptome rasch zu kontrollieren und weitere Infektionen $\mathrm{zu}$ vermeiden. Die systemisch wirksamen, oralen Antibiotika können jedoch zu Nebenwirkungen, Resistenzentwicklung und Interaktionen führen. Ärzte sollten dies - neben der Symptomlinderung und dem breiten antibakteriellem Wirkspektrum - bei der Auswahl des Antibiotikums mit berücksichtigen.
\end{abstract}

\section{Rifaximin}

Das lokale (topische) darmselektive Antibiotikum Xifaxan ${ }^{\circledR}$ (Rifaximin) erfüllt dieses Anforderungsprofil und ist in Deutschland seit 2008 zur Behandlung der nicht enteroinvasiv verlaufenden Reisediarrhö zugelassen. Diverse Studien konnten eine potente in-vitro-Aktivität gegenüber den meisten Erregern der Reisediarrhö belegen [1-5].

Wie bei anderen Antibiotika auch, kann eine Resistenz gegen Rifaximin entwickelt werden. Diese ist jedoch nur gering ausgeprägt und bleibt wegen seiner nicht plasmidvermittelten Codierung faktisch problemlos. Überdies sind die resistenten Keime schon kurze Zeit nach Therapieende nicht mehr im Stuhl nachweisbar, weil sie in Abwesenheit von Rifaximin einem Selektionsnachteil unterliegen [6].

Tab. 1 Nebenwirkungen (in \%) unter Rifaximin und Ciprofloxacin in einer randomisierten, doppeltblinden. kontrollierten Studie zur Reisediarrhö.

\begin{tabular}{|c|c|c|}
\hline $\begin{array}{l}\text { Rifaximin } \\
200 \mathrm{mg} \\
3-\mathrm{mal} / \mathrm{tgl} . \\
(\mathrm{n}=199)\end{array}$ & $\begin{array}{l}\text { Ciprofloxacin } \\
500 \mathrm{mg} \\
2-\mathrm{mal} / \mathrm{tgl} . \\
(\mathrm{n}=100)\end{array}$ & $\begin{array}{l}\text { Placebo } \\
2-\mathrm{mal} / \mathrm{tgl} \text {. } \\
(n=100)\end{array}$ \\
\hline & & \\
\hline
\end{tabular}

\begin{tabular}{|c|c|c|c|}
\hline $\begin{array}{l}\text { Jegliche } \\
\text { Neben- } \\
\text { wirkung }\end{array}$ & 26,6 & 24,0 & 25,0 \\
\hline Cephalgie & 8,0 & 5,0 & 9,0 \\
\hline Obstipation & 4,0 & 8,0 & 5,0 \\
\hline Flatulenz & 2,0 & 2,0 & 3,0 \\
\hline $\begin{array}{l}\text { Rektale } \\
\text { Tenesmen }\end{array}$ & 2,0 & 1,0 & 1,0 \\
\hline Schwindel & 1,0 & 2,0 & 2,0 \\
\hline Erbrechen & 1,0 & 2,0 & 1,0 \\
\hline Übelkeit & 0,5 & 2,0 & 1,0 \\
\hline
\end{tabular}

Therapie der Reisediarrhö

Die Wirksamkeit wurde in einer Reihe klinischer Studien an Patienten mit einer Reisediarrhö untersucht.

In einer doppelblinden Studie erhielten Probanden entweder 3 Tage lang Rifaximin (2-mal tgl. $400 \mathrm{mg}$ ) oder Ciprofloxacin (2-mal tgl. $500 \mathrm{mg}$ ). Die mediane Zeit vom Beginn der Behandlung bis zum letzten ungeformten Stuhl und die Zeit bis zur vollen Genesung war in beiden Gruppen vergleichbar [3].

Eine weitere multizentrische Studie verglich an 380 Probanden eine Behandlung mit Rifaximin (3-mal tgl. 200 oder $400 \mathrm{mg}$ ) oder mit Placebo. Die Behandlung dauerte 3 Tage. Die mittlere Dauer vom Beginn der Behandlung bis zum letzten ungeformten Stuhl war in beiden Rifaximingruppen deutlich niedriger als in der Placebogruppe. Vollständige Gesundung war in ähnlichem Verhältnis nach 5 Tagen in beiden Rifaximingruppen erreicht und damit der Placebogruppe deutlich überlegen [7].

Die Wirksamkeit einer Therapie mit Rifaximin (3-mal tgl. $200 \mathrm{mg}$ für 3 Tage) wurde mit Loperamid (initial $4 \mathrm{mg}$, dann $2 \mathrm{mg}$ nach jedem ungeformtem Stuhl) und Rifaximin plus Loperamid bei über 300 Probanden mit Reisediarrhö verglichen. Das Ergebnis: Eine alleinige Therapie mit Rifaximin oder eine Kombination beider Wirkstoffe war hinsichtlich der mittleren Dauer des letzten ungeformten Stuhlgangs beziehungsweise bis zur Genesung einer alleinigen Therapie mit Loperamid überlegen [8].

\section{Prävention der Reisediarrhö}

Auch die chemoprophylaktische Wirkung von Rifaximin wurde untersucht: Eine placebokontrollierte Studie mit $219 \mathrm{Me}-$ xikoreisenden ergab, dass Rifaximin (1-, 2- oder 3-mal tgl. $200 \mathrm{mg}$, über 14 Tage) versus Placebo die Inzidenz einer Reisediarrhö deutlich senkte [9].

\section{Verträglichkeitsprofil}

Rifaximin ist aufgrund der nahezu vollständig fehlenden Resorption extrem nebenwirkungsarm. Auftretende Nebenwirkungen sind meist nur gering ausgeprägt und unspezifisch (Tab. 1). In Studien zeigte Rifaximin hinsichtlich gastrointestina-

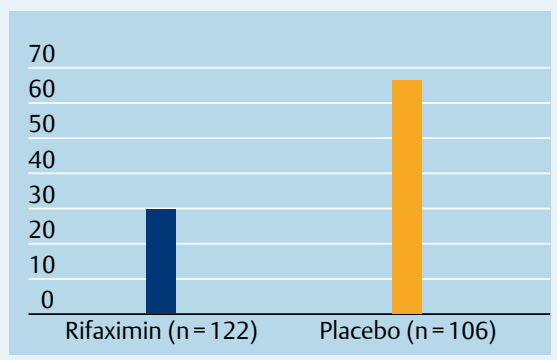

Abb. 1 Zeit bis zum letzten ungeformten Stuhl bei Patienten mit Reisediarrhö ohne Nachweis von pathogenen Keimen im Stuhl.

Quelle. mod. nach [11]

ler Nebenwirkungen und Cephalgien eine Verträglichkeit ähnlich wie Ciprofloxacin beziehungsweise Placebo [7, 10].

\section{Fazit}

Rifaximin ist ein praktisch nicht resorbiertes, ausschließlich topisch im Darmlumen wirksames, daher exzellent verträgliches Antibiotikum mit hoher Wirksamkeit gegen die meisten nicht invasiven Erreger einer Reisediarrhö. Die Substanz hat in mehreren Studien seine Überlegenheit gegenüber Placebo und Loperamid und seine ähnliche Wirksamkeit gegenüber systemisch wirksamen Antibiotika demonstriert und ist eine sinnvolle Ergänzung einer Reiseapotheke vor Aufenthalten in entsprechenden Risikogebieten.

Dr. Henrike Ruschewski, Dr. Viola Andresen,

Prof. Dr. Peter Layer, Hamburg

Quelle: Norgine GmbH, Marburg

Literatur

1 Ruiz J et al. Diagn Microbiol Infect Dis 2007; 59: 473-5

2 Sierra JM et al. Antimicrob Agents Chemother 2001; 45: 643-4

3 DuPont $\mathrm{HL}$ et al. Clin Infect Dis 2001; 33: 1807-15

4 Gomi H et al. Antimicrob Agents Chemother 2001; 45: 212-6

5 Hecht DW et al. Antimicrob Agents Chemother 2007; $51: 2716-9$

6 De Leo C et al. Drugs Exp Clin Res 1986; 12: 979-81

7 Steffen R et al. Am J Gastroenterol 2003; 98: 1073-8

8 DuPont HL et al. Clin Gastroenterol Hepatol 2007; 5: 451-6

9 DuPont HL et al. Ann Intern Med 2005; 142: 805-12

10 Taylor DN et al. Am J Trop Med Hyg 2006; 74: $1060-6$

11 DuPont H et al. J Travel Med 2007; 14: 16-9 


\section{Mehr Angebote und einen Apple iPad 2}

Anlässlich seines 25-jährigen Jubiläums hat das CRM Centrum für Reisemedizin sein Angebot CRM travel.NET erweitert und bietet für begrenzte Zeit ergänzend CRM travel.NET plus an: Zusätzlich zum Leistungs- und Servicepaket des Basisangebots haben Mitglieder von CRM travel.NET plus die Möglichkeit alle 2 Jahre an einem CRM Basis-Refresherseminar teilzunehmen und erhalten darüber hinaus ein Apple iPad 2 geschenkt.

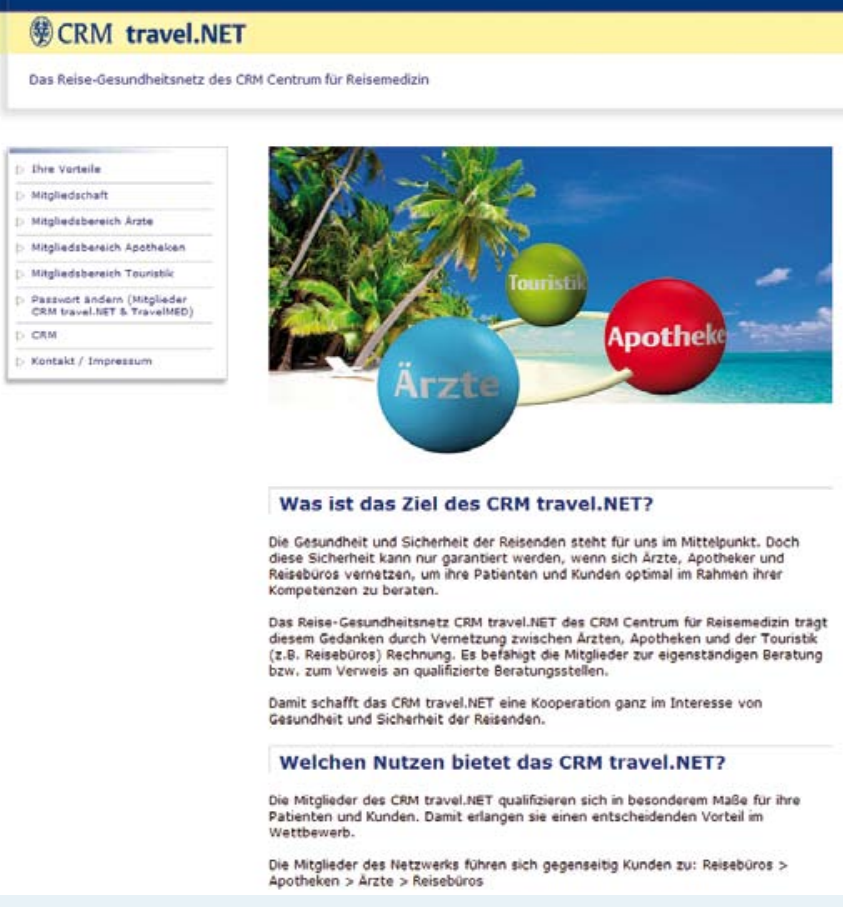

CRM travel.NET

Die größte reisemedizinische Community im deutschsprachigen Raum, ermöglicht Reisemedizinern, Apothekern sowie Mitarbeitern von Reisebüros sich zu vernetzen und zu aktuellen Themen der Reisemedizin auszutauschen. Der Dialog mit Partnern aus der Region bietet auch die Möglichkeit, Kunden und Patienten an geeignete Beratungsstellen zu verweisen und selbst neue Kundengruppen zu gewinnen.

Mitglieder des CRM travel.NET erhalten zudem ein umfangreiches Paket von Produkten und Serviceleistungen für ihre reisemedizinische Beratung: Die CRM Handbücher, der 14-tägige CRM-Infodienst, die Teilnahme an den CRM Online-Fortbildungen und vieles mehr. Das Jubiläumsangebot CRM travel.NET plus kostet für Ärzte und Apotheker 39,90Euro pro Monat bei einer Mindestvertragslaufzeit von 24 Monaten und ist bis zum 31.08.2013 verfügbar.

Eine Übersicht aller Produkte und Serviceleistungen, die CRM travel.NET und CRM travel.NET plus seinen Mitgliedern bieten, finden Interessierte unter http://travelnet.crm.de/index.html. 